\title{
Hybrid-Mode Multiple Access for UAV-BS Assisted Communications with UL-DL Rate Balancing
}

\author{
Haiyong Zeng*, Xu Zhu*†, Yufei Jiang*, Zhongxiang Wei*, and Yong Hao* \\ * School of Electronic and Information Engineering, Harbin Institute of Technology, Shenzhen, China \\ $\dagger$ School of Electrical Engineering and Electronics, University of Liverpool, Liverpool, UK \\ * School of Electrical Engineering and Electronics, University College London, London, UK \\ Emails: zenghaiyong@stu.hit.edu.cn,xuzhu@liverpool.ac.uk, jiangyufei@hit.edu.cn, \\ zhongxiang.wei@ucl.ac.uk, haoyong@stu.hit.edu.cn
}

\begin{abstract}
In this paper, we propose an unmanned aerial vehicle (UAV) base station (BS) assisted communication system for a special event (e.g., a football game) with heterogeneous traffic demands by all users and the uplink (UL)-downlink (DL) rate balancing requirement. With respect to UAV's high mobility, we propose a hybrid mode multiple access (HMMA) strategy where both orthogonal multiple access (OMA) and non-orthogonal multiple access (NOMA) techniques are utilized to meet heterogeneous traffic demands. Specifically, NOMA is utilized to achieve high average data rate, and OMA helps to meet the instantaneous rate demands of users. The proposed HMMA strategy has a high degree of freedom and provides superior minimum average rate across all users and a higher user fairness than the previous work with OMA only or NOMA only, where the instantaneous rate demands of users may not always be guaranteed during UAV's flight time due to dynamic channel changes, the interuser interference and successive interference cancellation (SIC) error propagation. Furthermore, to the best of our knowledge, this is the first work to investigate joint UL-DL optimization for a UAV assisted wireless system. Based on the channel reciprocity of the air-ground channels, an alternative algorithm is proposed to conduct joint UL-DL optimization of bandwidth assignment and UAV trajectory to accommodate heterogeneous rate demands across users and achieve quasi-balanced average rates in UL and DL.
\end{abstract}

Index Terms-UAV assisted communications, hybrid OMA/NOMA transmission, user fairness, UL-DL resource optimization

\section{INTRODUCTION}

Owing to high mobility, flexible deployment, low cost and high probability of providing line-of-sight (LoS) links, unmanned aerial vehicle (UAV) is emerging as an effective complementary to the fifth generation $(5 \mathrm{G})$ wireless communications and has been widely deployed as aerial base station (BS) to serve a group of ground users [1]-[3]. As stated in [3], the services provided by UAV-BS assisted systems can be mainly classified into two types: 1) delay-sensitive services where a minimum instantaneous rate is required to be guaranteed at any time over the service period of UAV, and 2) delay-tolerant services with no instantaneous rate constraint. For example, in a football stadium, the audiences share contents via online social networks, where some services such as video call are delay-sensitive and mission critical which require high instantaneous data rate, while some other services such as upload/download are delay-tolerant which just require high average data rate. Also, in this case, the requirements for uplink (UL) and downlink (DL) data rates are comparable [4], unlike a conventional wireless network where DL rate is normally much higher than UL rate [5].

In [3] and [6], a UAV assisted orthogonal frequency division multiple access (OFDMA) system was investigated, where the minimum throughput across all users is maximized by joint bandwidth assignment, power allocation and UAV trajectory design. In order to provide enhanced system spectrum efficiency (SE) performance and make full use of the underutilized wireless resources in orthogonality based approaches, powerdomain NOMA has been applied to UAV assisted systems [7][8], where a group of users share the same frequency band simultaneously with different power levels, and successive interference cancellation (SIC) can be utilized in signal detection, at the cost of increasing decoding complexity and multi-user interference. In [9] and [10], the effects of altitude or trajectory on energy efficiency and outage probability were studied for NOMA systems. The maximum average rate of NOMA that can be achieved by trajectory optimization was studied in [11], and it showed that the UAV assisted NOMA scheme envisions a superior throughput performance over the UAV assisted orthogonal multiple access (OMA) method. Although NOMA can provide a high average rate, the instantaneous rate demands of NOMA users may not always be guaranteed during UAV's flight time due to dynamic channel changes and the inter-user interference from users with higher channel conditions with the same NOMA group and error propagation introduced in the SIC process [12]. In [13] and [14], a hybrid OMA/NOMA terrestrial communication system was investigated, where dynamic mode switching between OMA and NOMA is utilized according to channel conditions. However, the work in [13] and [14] for terrestrial communications does not apply to a UAVBS assisted wireless system due to high correlations between air-ground channels. This motivates us to explore the UAV assisted hybrid multiple access systems and the corresponding resource optimization problems to meet the heterogeneous rate requirements of ground users.

Furthermore, the aforementioned work has considered DL optimization only, leaving the UL and DL rates asymmetric due to the different NOMA transmission principles for UL and DL. In UL NOMA, the power back-off strategy [7] is 
adopted, where the users with weaker channels are assigned less transmit power, which is opposite to the power allocation principle of DL NOMA [11]. Also, the UL rate of each user is affected by other users in the same NOMA group and is a non-convex function with respect to UAV's trajectory. Hence, the work on DL NOMA in [11] cannot be applied to joint ULDL optimization, and the issue of how to balance the UL and DL rates in a special event such as a football game remains an open challenge to address.

Motivated by the above open issues, we propose a UAV assisted communication system with hybrid mode multiple access (HMMA) and joint UL-DL optimization for a special event, where both mission critical and delay-tolerant services are demanded simultaneously by each user and the data rates for UL and DL are required to be quasi-balanced. Our contributions are summarized as follows:

1. With the HMMA strategy proposed, during UAV's flight time, both OMA and NOMA are utilized simultaneously to meet the heterogeneous traffic demands of users. Specifically, NOMA is utilized to support high average rate demands, and OMA helps to meet the instantaneous rate demands. With respect to UAV's high mobility, the proposed HMMA strategy can meet a wide range of heterogeneous traffic demands of all users with a high degree of freedom as it allows dynamic resource allocation under each multiple access mode. The proposed UAV assisted HMMA scheme provides superior minimum average rate performance and a better fairness among users, compared to the previous UAV assisted OMA [6] or NOMA [11], where the instantaneous rate demands of users may not always be guaranteed during the flight of UAV due to dynamic channel changes and the inter-user interference and error propagation introduced in the SIC process.

2. To the best of our knowledge, this is the first work to investigate joint UL and DL optimization for the UAV assisted systems in a special event where the average rates in UL and DL are required to be quasi-balanced. While as stated above, the optimization of NOMA in DL [11] cannot be applied to UL due to their different power allocation strategies. Thanks to the reciprocity of the air-ground channels, an alternative algorithm is proposed to conduct joint UL-DL optimization of bandwidth assignment and UAV trajectory design to accommodate the heterogeneous rate demands across users and achieve comparable average rates in UL and DL.

3. A comprehensive theoretical analysis is conducted to solved the non-convex joint UL-DL resource optimization problem, with the consideration of SIC error propagation. For the bandwidth assignment, it is proven that the non-convex problem can be transformed into a feasible one by assuming that the transmit power is equally allocated over the bandwidth. Moreover, successive convex optimization is adopted to solve the formulated non-convex UAV trajectory problem.

The rest of this paper is organized as follows. Section II introduces the system model and the problem formulation. In Section III, efficient solutions are proposed to solve the joint UL-DL resource optimization problem. Section VI presents numerical results. The conclusion is drawn in Section V.

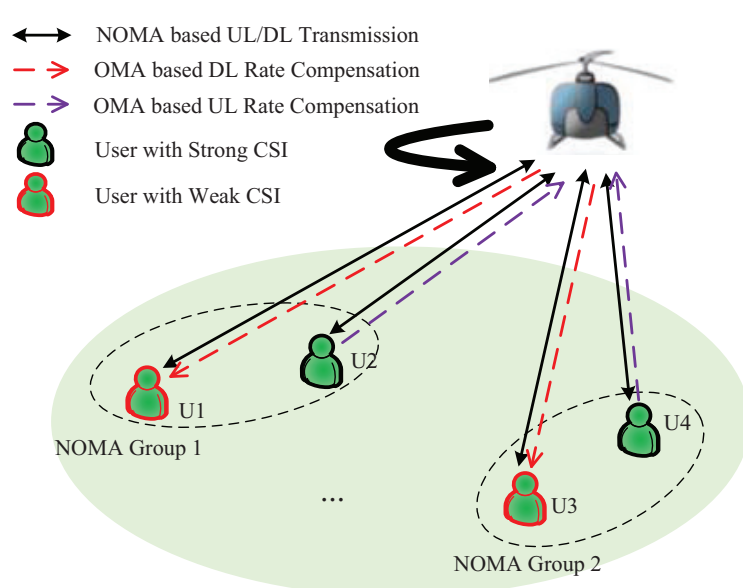

Fig. 1. System model of UAV assisted HMMA transmission.

\section{System Model}

We consider a UAV assisted system with $K$ ground users, where for each user hybrid OMA/NOMA scheme is utilized by the UAV BS to support heterogeneous rate demands across users in both UL and DL. As illustrated in Fig. 1, the $K$ ground users can be divided into $M$ groups, and the users in each group can communicate with the UAV BS by using NOMA strategy through dedicated scheduled bandwidth. Denote the set of users in group $m(m \in\{1, \ldots, M\})$ as $K_{m}$. Note that although NOMA enables multi-user multiplexing and provides a high system SE [1], the instantaneous rate demands of NOMA users may not always be guaranteed during UAV's flight time due to 1) dynamic channel changes and 2) the inter-user interference from users with higher channel conditions within the same NOMA group and error propagation introduced in the SIC process [12], especially for the users with lower channel conditions. Therefore, to assure instantaneous rate requirements across users, HMMA scheme can be utilized where a fraction of bandwidth can be scheduled to serve the users with poor performance in an OMA manner for rate compensation.

Assume the UAV flies at a fixed altitude of $H$ and flies above the terrestrial users within time length of $T$, which is divided into $N$ equally spaced time slots. The UAV's trajectory at time slot $n(n \in\{1,2 \ldots, N\})$ can be denoted as $\mathbf{q}[n]=$ $[x[n], y[n]]$, which should satisfy $\|\mathbf{q}[n+1]-\mathbf{q}[n]\| \leq S_{\max }$ and $\mathbf{q}[N]=\mathbf{q}[1]$, where $S_{\max }$ and $[x[n], y[n]]$ are respectively the maximum speed and the UAV coordinates. Assuming that the channel state information (CSI) is perfectly known at both UAV and users through feedback [6], and the Doppler effect has been perfectly compensated [1], then the air-ground link can be modeled as LoS. The channel power gain $h_{k, n}$ between user $k$ and UAV at time slot $n$ is $h_{k, n}=\frac{\gamma_{0}}{H^{2}+\left\|\mathbf{q}[n]-\mathbf{w}_{k}\right\|^{2}}$, with $\mathbf{w}_{k}$ and $\gamma_{0}$ denoting the coordinate and the channel power gain of user $k$ at the reference distance of $d_{0}=1 \mathrm{~m}$.

\section{A. Downlink HMMA Transmission}

For DL transmission, the received signal $r_{k, n}^{\mathrm{DL}}$ at an arbitrary user $k$ in NOMA group $m\left(k \in K_{m}\right)$ at time slot $n$ can be 
expressed as

$$
r_{k, n}^{\mathrm{DL}}=\sqrt{p_{k, n}^{\mathrm{DL}} h_{k, n}} s_{k, n}^{\mathrm{DL}}+\sum_{j \in K_{m}, j \neq k} \sqrt{p_{j, n}^{\mathrm{DL}} h_{k, n}} s_{j, n}^{\mathrm{DL}}+w^{\mathrm{DL}},
$$

where $p_{k, n}^{\mathrm{DL}}$ and $s_{k, n}^{\mathrm{DL}}$ denote the transmission power and the signal of user $k$ in DL, respectively. $w^{\mathrm{DL}}$ is the Gaussian noise. To make an attractive balance between decoding complexity and system performance, we assume the same time and frequency resource element is shared by two users [9]-[11]. It is noteworthy that the scheme for two-user multiplexing can be directly extended to the case with more users. Denote the indexes of users in NOMA group $m$ as $k_{1}$ and $k_{2}$, and we keep the users in each group fixed during UAV's flight time. Without loss of generality, we assume $h_{k_{1}, n}^{\mathrm{DL}} \leq h_{k_{2}, n}^{\mathrm{DL}}$, which indicates that user $k_{1}$ is weak user and user $k_{2}$ is strong user.

At the receiver, SIC is adopted to decode the superposed signals. Due to the imperfect SIC decoding, there exists residual power carried to the decoding of next level, which may cause error propagation [7]. Denote the bandwidth allocated to NOMA group $m$ for DL transmission as $B_{m, n}^{\mathrm{NO} D L}$, based on [7] and [11], the downlink NOMA rates of users $k_{1}$ and $k_{2}$ can be respectively given as

$$
\begin{gathered}
R_{k_{1}, \text { weak }}^{\mathrm{NO}}=B_{m, n}^{\mathrm{NO}, \mathrm{DL}} \log _{2}\left(1+\frac{(1-\theta) P_{m, n}^{\mathrm{DL}} h_{k_{1}, n}}{\theta P_{m, n}^{\mathrm{DL}} h_{k_{1}, n}+N_{0} B_{m, n}^{\mathrm{NO}, \mathrm{DL}}}\right), \\
R_{k_{2}, \text { strong }}^{\mathrm{NO}, \mathrm{DL}}=B_{m, n}^{\mathrm{NO}, \mathrm{DL}} \log _{2}\left(1+\frac{\theta P_{m, n}^{\mathrm{DL}} h_{k_{2}, n}}{\omega(1-\theta) P_{m, n}^{\mathrm{DL}} h_{k_{2}, n}+N_{0} B_{m, n}^{\mathrm{NO}, \mathrm{DL}}}\right),
\end{gathered}
$$

where $P_{m, n}^{\mathrm{DL}}$ and $\theta$ denote the downlink transmission power for NOMA group $m$ and the power allocation ratio between user $k_{1}$ and user $k_{2}$, respectively. $\omega$ is the proportion of SIC residual power and $N_{0}$ means the Gaussian noise spectral power density.

As mentioned above, to assure the DL instantaneous rate requirement of weak user $k_{1}$ which suffers from inter-user interference from strong user $k_{2}$ and enhance user fairness, a fraction of bandwidth $B_{m, n}^{\mathrm{O}, \mathrm{DL}}$ and transmission power $P_{m, n}^{\mathrm{O}, \mathrm{DL}}$ can be scheduled to serve user $k_{1}$ in an OMA manner to compensate instantaneous rate. Following [11], for fairness in NOMA and OMA performance comparison, the compensated rate of user $k_{1} R_{k_{1} \text {,weak }}^{\mathrm{O} \text {,DL }}$ can be obtained as

$$
R_{k_{1} \text {,weak }}^{\mathrm{O}, \mathrm{DL}}=\frac{1}{2} B_{m, n}^{\mathrm{O}, \mathrm{DL}} \log _{2}\left(1+\frac{P_{m, n}^{\mathrm{O}, \mathrm{DL}} h_{k_{1}, m}}{N_{0} B_{m, n}^{\mathrm{O}, \mathrm{DL}}}\right) .
$$

\section{B. Uplink HMMA Transmission}

For the UL transmission, the received superposed signal $r_{n}^{\mathrm{UL}}$ at UAV from NOMA group $m$ can be expressed as

$$
r_{n}^{\mathrm{UL}}=\sum_{j \in K_{m}} \sqrt{p_{j, n}^{\mathrm{UL}} h_{j, n}} s_{j, n}^{\mathrm{UL}}+w^{\mathrm{UL}}
$$

where $p_{j, n}^{\mathrm{UL}}$ and $s_{j, n}^{\mathrm{UL}}$ denote respectively the UL transmission power and the signal of user $j$ at time slot $n$.

For uplink SIC process, the signal of strong user $k_{2}$ is firstly decoded, after that the UAV removes it from the superposed signals and then decodes the signal of weak user $k_{1}$ [7]. In UL transmission, since a BS generally has much more capable and elaborate hardware than a user, we assume perfect SIC process at UAV-BS side [7]. Denote the bandwidth allocated to group $m$ for UL NOMA transmission as $B_{m, n}^{\mathrm{NO}, \mathrm{UL}}$, then the uplink NOMA rates of user $k_{1}$ and $k_{2}$ are given by

$$
\begin{gathered}
R_{k_{1}, \text { weak }}^{\mathrm{NO}, \mathrm{UL}}=B_{m, n}^{\mathrm{NO}, \mathrm{UL}} \log _{2}\left(1+\frac{\theta P_{m, n}^{\mathrm{UL}} h_{1, n}}{N_{0} B_{m, n}^{\mathrm{NO}, \mathrm{UL}}}\right), \\
R_{k_{2}, \text { strong }}^{\mathrm{NO}, \mathrm{UL}}=B_{m, n}^{\mathrm{NO}, \mathrm{UL}} \log _{2}\left(1+\frac{(1-\theta) P_{m, n}^{\mathrm{UL}} h_{2, n}}{\theta P_{m, n}^{\mathrm{UL}} h_{1, n}+N_{0} B_{m, n}^{\mathrm{NO}, \mathrm{UL}}}\right),
\end{gathered}
$$

where $P_{m, n}^{\mathrm{UL}}$ denotes the UL transmission power for NOMA group $m$. The sum rate of users in NOMA group $m R_{m, n}^{\mathrm{NO}, \mathrm{UL}}$ is

$$
R_{m, n}^{\mathrm{NO}, \mathrm{UL}}=B_{m, n}^{\mathrm{NO}} \log _{2}\left(1+\frac{\left(\theta h_{k_{1}, n}+(1-\theta) h_{k_{2}, n}\right) P_{m, n}^{\mathrm{UL}}}{N_{0} B_{m, n}^{\mathrm{NO}}}\right)
$$

Similarly, to guarantee UL instantaneous rate demands, a fraction of bandwidth $B_{m, n}^{\mathrm{O}, \mathrm{UL}}$ and transmission power $P_{m, n}^{\mathrm{O}, \mathrm{UL}}$ are scheduled to serve user $k_{2}$ which suffers from inter-user interference from user $k_{1}$. The uplink compensated rate of user $k_{2}$ at time slot $n$ can be expressed as

$$
R_{k_{2}, \text { strong }}^{\mathrm{O}, \mathrm{LL}}=\frac{1}{2} B_{m, n}^{\mathrm{O}, \mathrm{UL}} \log _{2}\left(1+\frac{P_{m, n}^{\mathrm{O}, \mathrm{UL}} h_{k_{2}, n}}{N_{0} B_{m, n}^{\mathrm{O}, \mathrm{UL}}}\right) .
$$

Let $\beta_{m, n}=1$ if $h_{k_{1}, n} \leq h_{k_{2}, n}$, otherwise $\beta_{m, n}=0$. Then the DL and UL achievable rates of user $k_{1}$ and $k_{2}$ in group $m$ at time slot $n$ can be respectively obtained as

$$
\begin{aligned}
& R_{k_{1}, n}^{\mathrm{DL}}=\beta_{m, n}\left(R_{k_{1}, \text { weak }}^{\mathrm{NO}}+R_{k_{1} \text {,weak }}^{\mathrm{O}, \mathrm{DL}}\right)+\left(1-\beta_{m, n}\right) R_{k_{1}, \text { strong }}^{\mathrm{NO}, \mathrm{DL}}, \\
& R_{k_{2}, n}^{\mathrm{DL}}=\beta_{m, n} R_{k_{2} \text {,strong }}^{\mathrm{NO}}+\left(1-\beta_{m, n}\right)\left(R_{k_{2} \text {,weak }}^{\mathrm{NO}}+R_{k_{2}, \text { weak }}^{\mathrm{O} \text {,DL }}\right) \text {, } \\
& R_{k_{1}, n}^{\mathrm{UL}}=\beta_{m, n} R_{1, \text { weak }}^{\mathrm{NO}}+\left(1-\beta_{m, n}\right)\left(R_{1, \text { strong }}^{\mathrm{NO}}+R_{k_{1}, \text { strong }}^{\mathrm{O}, \mathrm{UL}}\right), \\
& R_{k_{2}, n}^{\mathrm{UL}}=\beta_{m, n}\left(R_{2, \text { strong }}^{\mathrm{NO}}+R_{k_{2} \text {, strong }}^{\mathrm{O}, \mathrm{LL}}\right)+\left(1-\beta_{m, n}\right) R_{2, \text { weak }}^{\mathrm{NO} \text {. }}
\end{aligned}
$$

Based on (12) and (13), the overall sum rate of users in NOMA group $m R_{m, n}^{\text {group,UL }}$ can be written as

$$
R_{m, n}^{\text {group,UL }}=R_{m, n}^{\mathrm{NO}, \mathrm{UL}}+\beta_{m, n} R_{k_{2}, \text { strong }}^{\mathrm{O}, \mathrm{L}}+\left(1-\beta_{m, n}\right) R_{2, \text { weak }}^{\mathrm{O} \text {. }} .
$$

\section{Problem Formulation}

In this paper, we dedicate to maximizing the minimum average rate across all users in both UL and DL transmission for the proposed UAV assisted HMMA system, while guaranteeing instantaneous rate requirements of users.

Denote the minimum UL or DL average rate across all users as $\eta=\min _{i, k} \frac{1}{N} \sum_{n=1}^{N} R_{k, n}^{i}, k \in\{1,2, \ldots, K\}$, with $i$ as the indicator for UL or DL $i \in\{\mathrm{UL}, \mathrm{DL}\}$. Denote $\mathrm{Q}$ as the UAV trajectory matrix. Let $\mathbf{B}^{\mathrm{DL}}=\left\{\left[B_{m, n}^{\mathrm{DL}}\right]_{M \times N},\left[B_{m, n}^{\mathrm{O}, \mathrm{DL}}\right]_{M \times N}\right\}$ and $\mathbf{B}^{\mathrm{UL}}=\left\{\left[B_{m, n}^{\mathrm{NO}, \mathrm{UL}}\right]_{M \times N},\left[B_{m, n}^{\mathrm{O}, \mathrm{UL}}\right]_{M \times N}\right\}$ respectively denote the bandwidth assignment matrix for DL and UL. The bandwidth for DL and UL transmission can be expressed as $B_{n}^{\mathrm{DL}}=$ $\sum_{m=1}^{M}\left(B_{m, n}^{\mathrm{NO}, \mathrm{DL}}+B_{m, n}^{\mathrm{O}, \mathrm{DL}}\right)$ and $B_{n}^{\mathrm{UL}}=\sum_{m=1}^{M}\left(B_{m, n}^{\mathrm{NO}, \mathrm{UL}}+B_{m, n}^{\mathrm{O}, \mathrm{UL}}\right)$, respectively. For power allocation, we assume that the transmit power is equally allocated over the bandwidth. As a consequence, the power allocation can be simultaneously achieved through bandwidth assignment.

Base on the above analysis, the max-min problem for the UAV assisted HMMA system can be formulated as 


$$
\begin{array}{ll} 
& \mathbf{P 0} \max _{\mathbf{B}^{\mathrm{DL}}, \mathbf{B}^{\mathrm{UL}}, \mathbf{Q}} \eta \\
\text { s.t. } & \frac{1}{N} \sum_{n=1}^{N} R_{k, n}^{i} \geq \eta, \forall k, i \\
& R_{k, n}^{i} \geq \alpha_{k} \frac{1}{N} \sum_{n=1}^{N} R_{k, n}^{i}, \forall k, n, i \\
& B_{n}^{\mathrm{DL}}+B_{n}^{\mathrm{UL}} \leq B, \forall n \\
& \|\mathbf{q}[n+1]-\mathbf{q}[n]\| \leq S_{\max }, \\
& \mathbf{q}[N]=\mathbf{q}[1],
\end{array}
$$

where $(15 b)$ is the constraint for the average rate of users in $\mathrm{UL}$ and DL, (15c) is the instantaneous rate constraint for each user at each time slot in both links. Specifically, the concept of minimum-rate ratio (MRR) $\alpha$ is introduced to indicate the ratio of the required minimum data rate over the user's total average rate [6], which means that at any time slot, $\alpha$ fraction of its average rate over $N$ time slots is delay-sensitive and the remaining $(1-\alpha)$ fraction is delay-tolerant. (15d) indicates that the total utilizable bandwidth is limited to $B$. UAV trajectory is constrained by (15e) and (15f).

\section{Proposed SOLUTION}

Due to the non-convex users' rates constraints in (15b) and (15c), and the UAV trajectory constraints in (15e) and (15f), the optimization problem $\mathbf{P 0}$ is non-convex with respect to bandwidth $\mathbf{B}^{\mathrm{DL}}, \mathbf{B}^{\mathrm{UL}}$ and trajectory $\mathbf{Q}$. It is challenging to achieve the optimal solution to the considered problem in polynomial time. To this end, P0 can be decomposed into several sub-problems, namely bandwidth assignment and UAV trajectory design, and an alternative algorithm is proposed to jointly solve the sub-problems.

\section{A. Bandwidth Assignment}

With a given UAV trajectory $\mathbf{Q}$, the bandwidth assignment problem can be formulated as

$$
\begin{aligned}
& \text { P1.1 : } \max _{\mathbf{B}^{\mathrm{DL}}, \mathbf{B}^{\mathrm{UL}}} \eta \\
& \text { s.t. } \quad(15 b)-(15 d),
\end{aligned}
$$

which is still non-convex with respect to $\mathbf{B}^{\mathrm{DL}}, \mathbf{B}^{\mathrm{UL}}$.

In the following, proposition 1 is presented to transform problem P1.1 into a more feasible form.

proposition1: In UAV assisted HMMA systems, given that the transmit power is equally allocated over the bandwidth, problem P1.1 can be transformed into a convex form as

$$
\begin{array}{ll} 
& \mathbf{P} 1.2 \max _{\mathbf{B}^{\mathrm{DL}}, \mathbf{B}^{\mathrm{UL}}} \eta \\
\text { s.t. } & \frac{1}{N} \sum_{n=1}^{N} R_{k, n}^{\mathrm{group}, \mathrm{UL}} \geq 2 \eta, \forall k, \\
& \frac{1}{N} \sum_{n=1}^{N} R_{k, n}^{\mathrm{DL}} \geq \eta, \forall k, \\
& R_{k, n}^{\mathrm{UL}} \geq \alpha_{k} \eta, \forall k, n, \\
& R_{k, n}^{\mathrm{DL}} \geq \alpha_{k} \eta, \forall k, n, \\
& B_{n}^{\mathrm{DL}}+B_{n}^{\mathrm{UL}} \leq B, \forall n .
\end{array}
$$

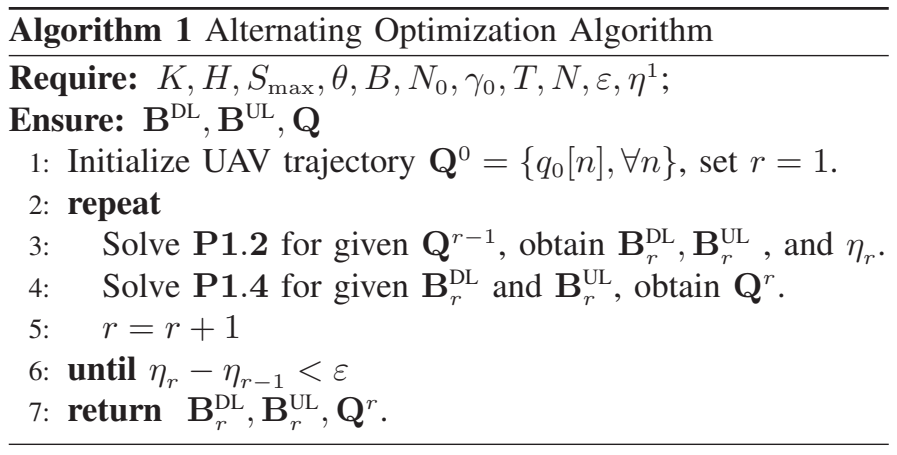

proof of proposition1: First, since the transmit power for both DL and UL is equally allocated over the bandwidth, we have $P_{t}^{i} / B_{n}^{i}=P_{m, n}^{\mathrm{NO}, i} / B_{m, n}^{\mathrm{NO}, i}=P_{m, n}^{\mathrm{O}, i} / B_{m, n}^{\mathrm{O}, i}$, with $P_{t}^{i}(i \in$ $\{\mathrm{DL}, \mathrm{UL}\})$ denoting the transmit power for DL/UL transmission. Substituting the above equalities into (2)-(4) yields

$$
\begin{gathered}
R_{k_{1}, \text { weak }}^{\mathrm{NO}, \mathrm{DL}}=B_{m, n}^{\mathrm{NO}, \mathrm{DL}} \log _{2}\left(1+\frac{(1-\theta) P_{t}^{\mathrm{DL}} h_{k_{1}, n}}{\theta P_{t}^{\mathrm{DL}} h_{k_{1}, n}+N_{0} B}\right) \\
R_{k_{2} \text {,strong }}^{\mathrm{NO}, \mathrm{DL}}=B_{m, n}^{\mathrm{NO}, \mathrm{DL}} \log _{2}\left(1+\frac{\theta P_{t}^{\mathrm{DL}} h_{k_{2}, n}}{\omega(1-\theta) P_{t}^{\mathrm{DL}} h_{k_{2}, n}+N_{0} B}\right) \\
R_{k_{1}, \text { weak }}^{\mathrm{O} \text {,DL }}=\frac{1}{2} B_{m, n}^{\mathrm{O}, \mathrm{DL}} \log _{2}\left(1+\frac{P_{t}^{\mathrm{DL}} h_{k_{1}, m}}{N_{0} B}\right)
\end{gathered}
$$

which are convex with respect to $\mathbf{B}^{\mathrm{DL}}$. As a result, we obtain that constraint (16c) in $\mathbf{P} \mathbf{1 . 2}$ is convex with respect to $\mathbf{B}^{\mathrm{DL}}$. Using the similar derivation, we can also prove that constraint (16b) is convex with respect to $\mathbf{B}^{\mathrm{UL}}$. Then, it can be learned from (16d) and (16e) that the feasible solution of P1.1 is generally a subset of that of $\mathbf{P 1 . 2}$, and the problems $\mathbf{P} \mathbf{1 . 1}$ and $\mathbf{P 1 . 2}$ are equivalent if all of the ground users can achieve the equal average rate when obtaining the optimal solution to P1.2 [6]. Based on the above transformation, the solution to the original bandwidth assignment problem P1.1 can be obtained by solving the convex problem P1.2 using CVX.

\section{B. UAV Trajectory Design}

Now the bandwidth assignment have been obtained, we then attempt to design the UAV trajectory. The UAV trajectory problem for the UAV assisted HMMA systems is

$$
\begin{aligned}
& \text { P1.3 }: \max _{\mathbf{Q}} \eta \\
& \text { s.t. }(15 e),(15 f) \text {, and }(16 \mathrm{~b})-(16 \mathrm{e}),
\end{aligned}
$$

which is non-convex. To this end, successive convex optimization is utilized to solve the problem.

First, for DL transmission, though the rate of users in (10) and (11) are non-convex with respect to $\mathbf{q}[n]$, they are convex functions of $\left\|\mathbf{q}[n]-\mathbf{w}_{k}\right\|^{2}$. As a result, we can use the firstorder Taylor's series expansion as the lower bound of the original problem to achieve the approximation [1]. On the other hand, the UL NOMA rate in (7) is not convex with respect to $\left\|\mathbf{q}[n]-\mathbf{w}_{k}\right\|^{2}$ and cannot be approximated by the first-order Taylor's series expansion due to the inter-user interference in the denominator. Note that the sum rate of users in each NOMA group $m R_{m, n}^{\text {group,UL }}$ in (8) can be approximated as a 


$$
\begin{aligned}
& R_{k_{1}, n}^{\mathrm{DL}, \mathrm{lb}}=G_{k_{1}, n}^{\mathrm{DL}}+\beta_{m, n}\left(\frac{-B_{m, n}^{\mathrm{O}, \mathrm{UL}} P_{m, n}^{\mathrm{O}, \mathrm{DL}} \gamma_{0} \log _{2} e}{2\left(H^{2}+\left\|q^{r}[n]-w_{m, n, k_{1}}\right\|^{2}\right)\left(N_{0} B_{m, n}^{\mathrm{O}, \mathrm{DL}}\left(H^{2}+\left\|q^{r}[n]-w_{m, n, k_{1}}\right\|^{2}\right)+P_{m, n}^{\mathrm{O}, \mathrm{DL}} \gamma_{0}\right)}+\right. \\
& \left.\frac{-B_{m, n}^{\mathrm{NO}, \mathrm{UL}}(1-\theta) P_{m, n}^{\mathrm{NO}, \mathrm{DL}} \gamma_{0} \log _{2} e}{\left(N_{0} B_{m, n}^{\mathrm{NO}, \mathrm{DL}}\left(H^{2}+\left\|q^{r}[n]-w_{m, n, k_{1}}\right\|^{2}\right)+\theta P_{m, n}^{\mathrm{NO}, \mathrm{DL}} \gamma_{0}\right)\left(N_{0} B_{m, n}^{\mathrm{NO}, \mathrm{DL}}\left(H^{2}+\left\|q^{r}[n]-w_{m, n, k_{1}}\right\|^{2}\right)+P_{m, n}^{\mathrm{NO}, \mathrm{DL}} \gamma_{0}\right)}\right)+\left(1-\beta_{m, n}\right) \times \\
& \left(\frac{-B_{m, n}^{\mathrm{NO}, \mathrm{UL}} \log _{2} e \times \theta P_{m, n}^{\mathrm{NO}, \mathrm{DL}} \gamma_{0}}{\left(N_{0} B_{m, n}^{\mathrm{NO}, \mathrm{DL}}\left(H^{2}+\left\|q^{r}[n]-w_{m, n, k_{1}}\right\|^{2}\right)+\omega(1-\theta) P_{m, n}^{\mathrm{No}, \mathrm{DL}}\right)\left(N_{0} B_{m, n}^{\mathrm{NO}, \mathrm{DL}}\left(H^{2}+\left\|q^{r}[n]-w_{m, n, k_{1}}\right\|^{2}\right)+(\omega(1-\theta)+\theta) P_{m, n}^{\mathrm{NO}, \mathrm{DL}} \gamma_{0}\right)}\right) \\
& G_{k_{1}, n}^{\mathrm{DL}}=\beta_{m, n}\left(B_{m, n}^{\mathrm{NO}, \mathrm{UL}} \log _{2}\left(1+\frac{(1-\theta) P_{m, n}^{\mathrm{No}, \mathrm{DL}} \gamma_{0}}{N_{0} B_{m, n}^{\mathrm{NO}, \mathrm{DL}}\left(H^{2}+\left\|q^{r}[n]-w_{m, n, k_{1}}\right\|^{2}\right)+\theta P_{m, n}^{\mathrm{NO}, \mathrm{DL}} \gamma_{0}}\right)+\frac{1}{2} B_{m, n}^{\mathrm{O}, \mathrm{UL}} \log _{2}(1+\right. \\
& \left.\left.\frac{P_{m, n}^{\mathrm{O}, \mathrm{DL}} \gamma_{0} /\left(N_{0} B_{m, n}^{\mathrm{O}, \mathrm{DL}}\right)}{H^{2}+\left\|q^{r}[n]-w_{m, n, k_{1}}\right\|^{2}}\right)\right)+\left(1-\beta_{m, n}\right) B_{m, n}^{\mathrm{NO}, \mathrm{UL}} \log _{2}\left(1+\frac{\theta P_{m, n}^{\mathrm{NO}, \mathrm{DL}} \gamma_{0}}{N_{0} B_{m, n}^{\mathrm{NO}, \mathrm{DL}}\left(H^{2}+\left\|q^{r}[n]-w_{m, n, k_{1}}\right\|^{2}\right)+\omega(1-\theta) P_{m, n}^{\mathrm{NO}, \mathrm{DL}} \gamma_{0}}\right)
\end{aligned}
$$

convex function of $\left\|\mathbf{q}[n]-\mathbf{w}_{k}\right\|^{2}$. As a result, the original UAV trajectory problem can be approximated as

$$
\begin{array}{ll} 
& \mathbf{P} 1.4: \max _{\mathbf{Q}} \eta \\
\text { s.t. } & \frac{1}{N} \sum_{n=1}^{N} R_{m, n}^{\text {group,UL,lb }} \geq 2 \eta, \forall m, n, \\
& \frac{1}{N} \sum_{n=1}^{N} R_{k, n}^{\mathrm{DL}, \mathrm{lb}} \geq \eta, \forall k, n, \\
& R_{k, n}^{\text {group,UL,lb }} \geq 2 \beta_{k} \eta, \forall k, n, \\
& R_{k, n}^{\mathrm{DL}, \mathrm{lb}} \geq \beta_{k} \eta, \forall k, n, \\
& (15 e),(15 f),
\end{array}
$$

where the expression of $R_{k_{1}, n}^{\mathrm{DL}, \mathrm{b}}$ is given in (21). Through the similar derivations of $R_{k_{1}, n}^{\mathrm{DL}, \mathrm{h}}$, the expression of $R_{k, n}^{\text {group,UL,lb }}$ can also be obtained. The approximated problem P1.4 is a convex quadratic problem and can be solved by CVX.

By alternately solving bandwidth assignment in P1.2 and trajectory design in P1.4, the original problem can be solved. The overall algorithm is concluded in Algorithm 1.

\section{Numerical Results}

In this section, numerical results are carried out to demonstrate the superiority of the proposed UAV assisted HMMA system. The altitude of UAV is $H=100 \mathrm{~m}$ and the maximum speed of UAV is $50 \mathrm{~m} / \mathrm{s}$. Users are distributed in the area of $1.5 \mathrm{~km} \times 1.5 \mathrm{~km}$. The total system bandwidth is $B=2$ $\mathrm{MHz}$. The transmission power $P_{t}^{\mathrm{UL}}$ and $P_{t}^{\mathrm{DL}}$ are $0.5 \mathrm{~W}$ and $0.1 \mathrm{~W}$, respectively. In addition, we set $N_{0}=-110 \mathrm{dBm} / \mathrm{Hz}$, $\gamma_{0}=-50 \mathrm{~dB}, \varepsilon=10^{-3}, T=50 \mathrm{~s}, K=6$ and $\theta=0.2$ [11].

Fig. 2 illustrates the impact of MRR $\alpha$ on the minimum average rate across users $\eta$ for various UAV assisted systems. As can be seen, the minimum average rate $\eta$ decreases as $\alpha$ grows, and the proposed UAV assisted HMMA scheme demonstrates a much higher $\eta$ than the previous UAV assisted NOMA [11] or OMA [6] methods, especially when $\alpha$ is in a relatively high level. For example, when $\alpha=0.8$, the proposed HMMA scheme achieves more than $25 \%$ and $81 \%$ higher $\eta$ over the previous NOMA [11] and OMA [6] schemes. The reason is that, the proposed HMMA scheme has a higher

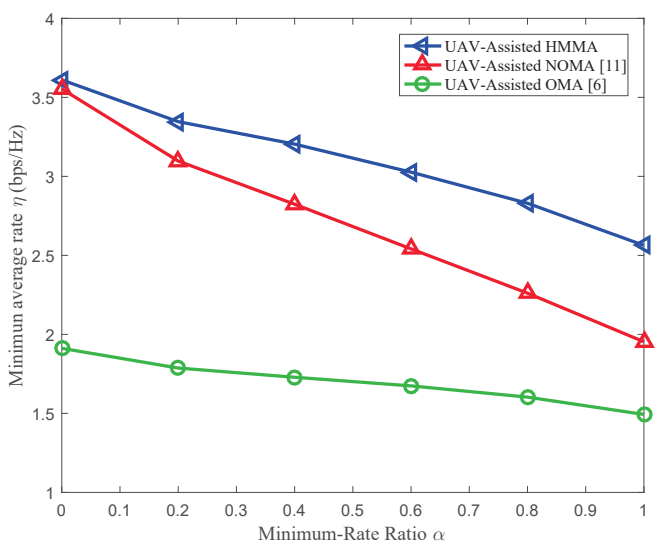

Fig. 2. Impact of the MMR $\alpha$ on the minimum average rate among users $\eta$.
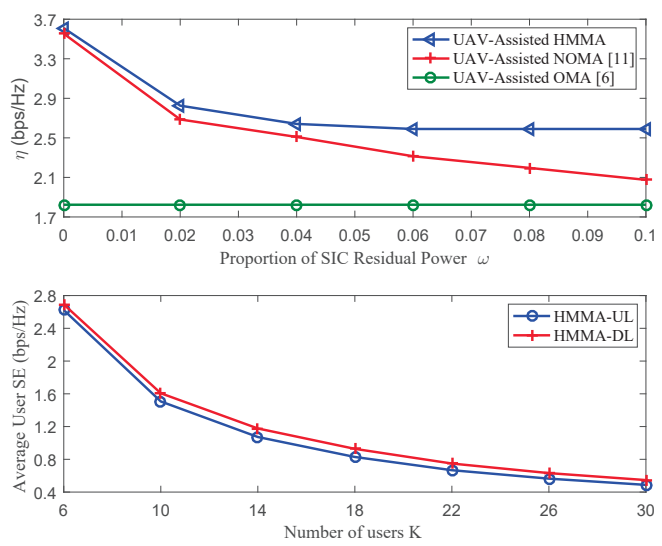

Fig. 3. a) Impact of the imperfect SIC on the minimum average rate among users $\eta$; b) Average user SE in UL and DL with different number of users $K$.

degree of freedom in dynamic resource allocation under each multiple access mode (OMA/NOMA), and a dedicated fraction of bandwidth can be scheduled to compensate the rate of users with poor performance in each NOMA group, which leads to superior minimum average rate and higher user fairness. 

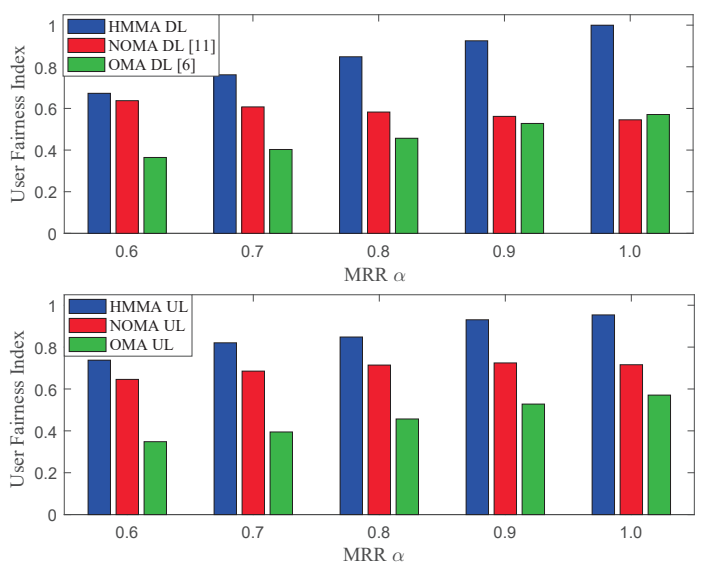

Fig. 4. User fairness index of different UAV assisted communication systems.

Fig. 3 (a) shows the impact of SIC residual power on $\eta$, with $\alpha=0$. As can be seen, the proposed HMMA scheme shows a better performance and is more robust against SIC error propagation than NOMA method. The average user SE in UL and DL under different number of users is shown in Fig. 3 (b), with MMR $\alpha=1$ and flight time $T=150 \mathrm{~s}$. It can be seen that the average user SE decreases with the number of users due to limited spectrum resources, and HMMA can achieve comparable average rates in UL and DL to meet the throughput balancing requirement of the special event.

In Fig. 4, user fairness index is presented to indicate the fairness among users under different MRR $\alpha$, with $T=50$ s. To make this a fair comparison among different UAV assisted systems, the user fairness index can be expressed as $J^{j}=\frac{\eta^{j}}{\sum \eta^{j}} \sum_{n=1}^{N}\left(\sum_{k=1}^{K} R_{k, n}\right)^{2} /\left(N \sum_{n=1}^{N} \sum_{k=1}^{K} R_{k, n}^{2}\right)$, with $\frac{\eta^{j}}{\sum \eta^{j}}$ denoting the normalized minimum average rate of mode $j$, $j \in\{$ HMMA,NOMA,OMA $\}$. It can be seen from Fig. 4 that the proposed HMMA scheme demonstrates a higher user fairness over the NOMA or OMA systems, especially with stringent instantaneous rate demands.

Fig. 5 illustrates the trajectories of the proposed UAV assisted HMMA systems, with different flight time $T$ and MRR $\alpha$. When the flight time is short, the UAV mainly flies in the central area due to maximum speed limitation. With a longer flight time, the UAV tends to fly closer to each user to enable a better LoS communication link. In addition, given a higher $\alpha$ (e.g., $T=150 \mathrm{~s}$ and $\alpha=0.9$ ), the instantaneous rate requirements of users become stringent, as a result, the UAV adjusts its trajectory to fly around all the users so that the heterogeneous traffic demands across users can be guaranteed.

\section{CONClusion}

In this paper, we have proposed a HMMA scheme for the UAV assisted system where both OMA and NOMA are utilized simultaneously to meet users' instantaneous and continuous traffic demands as well as UL-DL rate balancing requirement. With a stringent instantaneous rate demand across users, the proposed HMMA achieves more than $25 \%$ and $80 \%$ higher

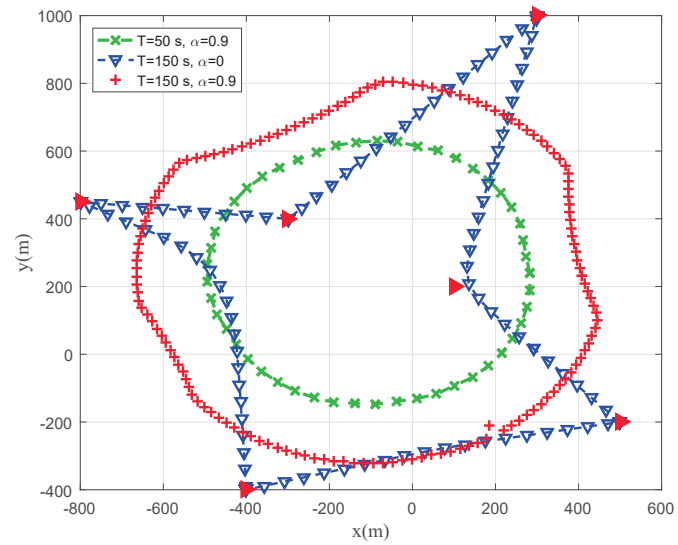

Fig. 5. UAV trajectories of the HMMA scheme with different $\alpha$ and $T$.

minimum average rate than the previous NOMA [11] and OMA [6] methods. In addition, joint UL-DL optimization is conducted to achieve comparable average UL and DL throughputs for the kind of special events considered in this paper.

\section{REFERENCES}

[1] Y. Liu, Z. Qin, Y. Cai, Y. Gao, G. Y. Li, and A. Nallanathan, "UAV Communications based on Non-orthogonal Multiple Access" IEEE Wireless Communi., vol. 26, no. 1, pp. 52-57, Feb. 2019.

[2] N. Zhao, F. Cheng, F. R. Yu, J. Tang, Y. Chen, G. Gui, and H. Sari, "Caching UAV Assisted Secure Transmission in Hyper-Dense Networks based on Interference Alignment," IEEE Trans. on Commun., vol. 51, no. 58, pp. 2281-2294, May 2018.

[3] Q. Wu and R. Zhang, "Delay-Constrained Throughput Maximization in UAV-enabled OFDM Systems," in Proc. IEEE APCC' 17, Perth, WA, Australia, Mar. 2018.

[4] I. Bergel, Y. Perets, and S. Shamai, "Uplink Downlink Rate Balancing in Cooperating Cellular Networks," IEEE Trans. Sig. Process., vol. 63, no. 23 , pp. 6272-6284, Dec. 2015.

[5] L. Zhang, W. Nie, G. Feng, F. Zheng, and S. Qin, "Uplink Performance Improvement by Decoupling Uplink/Downlink Access in HetNets,' IEEE Trans. Veh. Technol., vol. 66, no. 8, pp. 6862-6876, Aug. 2017.

[6] Q. Wu and R. Zhang, "Common Throughput Maximization in UAVEnabled OFDMA Systems with Delay Consideration," IEEE Trans. Commun., vol. 66, no. 12, pp. 6614-6627, Dec. 2018.

[7] Z. Zhang, H. Sun, and R. Q. Hu, "Downlink and Uplink Non-Orthogonal Multiple Access in a Dense Wireless Network," IEEE J. Sel. Areas Commun., vol. 35, no. 12, pp. 2771-2784, Dec. 2017.

[8] M. S. Ali, E. Hossain, A. A. Dweik, and D. I. Kim, "Downlink power allocation for CoMP-NOMA in multi-cell networks," IEEE Trans. on Commun., vol. 66, no. 9, pp. 3982-3998, Sep. 2018.

[9] M. F. Sohail, C. Y. Leow, and S. Won, "Non-Orthogonal Multiple Access for Unmanned Aerial Vehicle Assisted Communication," IEEE Access, vol. 6, pp. 22716-22727, 2018.

[10] P. K. Sharma and D. I. Kim, "UAV-Enabled Downlink Wireless System with Non-Orthogonal Multiple Access," in Proc. IEEE Globecom Workshops 2017, Singapore, 2017, pp. 1-6.

[11] J. Sun, Z. Wang, and Q. Huang, "Cyclical NOMA Based UAV-Enabled Wireless Network," IEEE Access, vol. 7, pp. 4248-4259, 2019.

[12] M. Zeng, A. Yadav, O. A. Dobre and H. V. Poor, "Energy-Efficient Joint User-RB Association and Power Allocation for Uplink Hybrid NOMAOMA," IEEE Internet of Things Journal, vol. 6, no. 3, pp. 5119-5131, June 2019.

[13] A. S. Marcano and H. L. Christiansen, "A Novel Method for Improving the Capacity in 5G Mobile Networks Combining NOMA and OMA," in Proc. IEEE VTC-Spring 2017, Sydney, Australia, 2017, pp. 1-5.

[14] Z. Song, Q. Ni, and X. Sun, "Spectrum and Energy Efficient Resource Allocation with QoS Requirements for Hybrid MC-NOMA 5G Systems," IEEE Access, vol. 6, pp. 37055-37069, Jul. 2018. 